\title{
Aerobic exercise improves cardiac function in rats with chronic heart failure through inhibition of the long non-coding RNA metastasis-associated lung adenocarcinoma transcript 1 (MALAT1)
}

\author{
Ling $\mathrm{Hu}^{1 \#}$, Ya-Nan Xü ${ }^{2 \#}$, Qian Wang ${ }^{3}$, Mei-Jie Liu ${ }^{4}$, Ping Zhang ${ }^{5}$, Lan-Ting Zhao ${ }^{5}$, Fang Liu ${ }^{5}$, \\ Dong-Yan $\mathrm{Zhao}^{2}$, He-Nan $\mathrm{Pei}^{3}$, Xing-Bao Yao ${ }^{6}$, Hua-Gang $\mathrm{Hu}^{7}$ \\ ${ }^{1}$ Department of Internal Medicine, Beijing Xiaotangshan Hospital, Beijing, China; ${ }^{2}$ Department of Cardiopulmonary Rehabilitation, Beijing \\ Xiaotangshan Hospital, Beijing, China; ${ }^{3}$ Department of Sports Rehabilitation, Beijing Xiaotangshan Hospital, Beijing, China; ${ }^{4}$ Medical Experiment \\ Center, China Academy of Chinese Medical Sciences, Beijing, China; ${ }^{5}$ Department of Cardiology, Beijing Tsinghua Changgung Hospital affiliated \\ with Tsinghua University, Beijing, China; ${ }^{6}$ Department of Sports Injury, Hebei Provincial Hospital of Traditional Chinese Medicine, Shijiazhuang, \\ China; ${ }^{7}$ Research Office, Beijing Xiaotangshan Hospital, Beijing, China \\ Contributions: (I) Conception and design: L Hu, YN Xu; (II) Administrative support: X.B Yao, HG Hu; (III) Provision of study materials or patients: \\ Q Wang, MJ Liu; (IV) Collection and assembly of data: P Zhang, LT Zhao; (V) Data analysis and interpretation: DY Zhao, F Liu, HN Pei; (VI) \\ Manuscript writing: All authors; (VII) Final approval of manuscript: All authors. \\ \#These authors contributed equally to this study. \\ Correspondence to: Dr. Hua-Gang Hu. Research Office, Beijing Xiaotangshan Hospital, No. 390, Wenquan Street, Beijing 102211, China. \\ Email: zthh1340@163.com; Dr. Xing-Bao Yao. Department of Sports Injury, Hebei Provincial Hospital of Traditional Chinese Medicine, No. 389, \\ Zhongshan East Road, Shijiazhuang 050011, China. Email: arthur0105@126.com.
}

Background: To explore the beneficial effects and underlying mechanisms of aerobic exercise on chronic heart failure (CHF).

Methods: A CHF rat model was induced via left anterior descending coronary artery ligation. Four weeks post-surgery, $\mathrm{CHF}$ rats received aerobic exercise training over an 8-week period and cardiac function indexes including xxx were analyzed. To investigate the mechanisms involved in the aerobic exercise-induced benefits on CHF, overexpression of the long non-coding RNA MALAT1 was examined both in vivo and in vitro. Furthermore, the interaction between MALAT1 and the microRNA miR-150-5p and the downstream PI3K/ Akt signaling pathway was investigated.

Results: Compared to the control group, the CHF rats showed evidence of left ventricular dysfunction including aggravated cardiac function indexes and lung to body weight ratio. The Masson staining demonstrated a significant degree of blue-stained fibrotic myocardial tissue in CHF rats compared to control rats. Furthermore, the levels of collagen I and collagen II were also markedly increased in CHF rats. Aerobic exercise improved cardiac function and left ventricular remodeling in rats with CHF. There was a significant reduction in the levels of the reactive oxygen species (ROS), inflammatory cytokines including TNF- $\alpha$, IL-6, and IL-1 $\beta$, and inflammatory mediums containing the matrix metalloproteinases (MMPs) MMP-2 and MMP-9. Moreover, CHF rats receiving aerobic exercise showed decreased myocardial apoptosis and increased expression of autophagy-related proteins including beclin-1 and LC3B-II. Overexpression of the lncRNA MALAT1 eliminated all the beneficial effects related to aerobic exercise in CHF rats. Subsequent investigations demonstrated that miR-150-5p expression was up-regulated in CHF-Tr rats and downregulated in CHF-Tr-MALAT1 rats. Furthermore, the downstream PI3K/Akt signaling pathway was re-activated in CHF-Tr-MALAT1 rats. In vitro experiments revealed that overexpression of MALAT1 reduced the miR-150-5p levels, resulting in increased cellular apoptosis and less autophagy. In addition, overexpression of MALAT1 suppressed the downstream PI3K/Akt signaling pathway. Restoring miR-150-5p 
level with a miR-150-5p mimic decreased the cellular apoptosis and increased autophagy, and the downstream PI3K/Akt signaling pathway was re-activated.

Conclusions: Aerobic exercise improved cardiac function through inhibition of the lncRNA MALAT1 in $\mathrm{CHF}$, and the potential mechanisms may be mediated via the miR-150-5p/PI3K/Akt signaling pathway.

Keywords: Aerobic exercise; chronic heart failure (CHF); metastasis-associated lung adenocarcinoma transcript 1

Submitted Dec 08, 2020. Accepted for publication Jan 29, 2021.

doi: 10.21037/atm-20-8250

View this article at: http://dx.doi.org/10.21037/atm-20-8250

\section{Introduction}

Chronic heart failure (CHF) is a common endpoint of various cardiovascular diseases, and seriously affects the prognosis of patients. The clinical features of CHF mainly include dyspnea, fatigue, decreased exercise tolerance caused by reduced cardiac output, skeletal muscle disease, and endothelial cell (EC) dysfunction (1). Inflammation and the production of reactive oxygen species (ROS) are characteristics of cardiac dysfunction and play an important role in the pathogenesis of CHF (2). In addition, insufficient cardiac output and vasoconstriction caused by activation of the sympathetic system can lead to tissue hypoxia, further increasing the production of oxygen free radicals (3). ROS are by-products of oxygen metabolism. It can directly react with proteins, nucleic acids, and other substances, leading to cell dysfunction and cell death through apoptosis and necrosis (4). In addition, the sustained release of ROS and the presence of inflammation can lead to cardiomyocyte hypertrophy, and ultimately the activation of matrix metalloproteinases (MMPs), collagen deposition, and myocardial fibrosis, all of which culminate in the progression to CHF (5).

Aerobic exercise refers to the rhythmic exercise performed by the human body under the condition of sufficient oxygen supply. At present, it is divided into lowintensity aerobic exercise and high-intensity intermittent aerobic exercise (HIIT) (6). The purpose of aerobic exercise is to enhance the endurance of the heart and lungs, resulting in adaptive changes in related systems and muscles (7). Researchers have reported that longterm moderate-intensity aerobic exercise can increase the production of nitric oxide (NO) by ECs, thereby increasing the vasodilation tension (8). de Beer et al. (9) confirmed that moderate-intensity aerobic exercise reduced coronary vascular tension and endothelin (ET) production, thereby increasing the blood flow reserves. Moderate aerobic exercise was shown to significantly reduce the levels of hypersensitive $\mathrm{C}$-reactive protein (CRP) in mouse plasma. Furthermore, aerobic exercise inhibited the secretion of monocyte chemotactic factor-1 (MCP-1) and adhesion factors of ECs, thereby suppressing the differentiation of monocytes into macrophages (10). Recently, a study reported that long-term aerobic exercise significantly up-regulated the expression of irisin in cardiomyocytes, which reduced the oxidative stress and inhibited the production of ROS. The potential mechanisms may involve improvement of the mitochondrial network structure to protect mitochondrial function (11).

Long non-coding RNAs (lncRNAs) are a group of noncoding RNAs composed of more than 200 nucleotides. Although lncRNAs cannot be translated into protein, they can affect gene function by regulating the process of transcription and translation (12). Studies have found that lncRNAs can bind directly to microRNAs (miRNAs) to regulate the expression levels of miRNA, thereby regulating gene function (13). The lncRNA metastasis-related lung adenocarcinoma transcript 1 (MALAT1) was first reported in non-small cell lung cancer and was positively correlated with poor prognosis (14). In recent years, the function of MALAT1 has been reported in a variety of tumors, but its role in cardiovascular disease has not yet been fully elucidated. Studies have shown that MALAT1 can improve left ventricular function in rats via reducing cardiomyocyte apoptosis (15). In addition, MALAT1 has been shown to participate in disease progression by regulating the autophagy of cardiomyocytes in reperfusion injury after acute myocardial infarction (AMI) (16). In the present report, a rat model was used to study the potential benefits of aerobic exercise on $\mathrm{CHF}$, and the possible mechanisms involving the lncRNA MALAT1 and the miR-150-5p/ $\mathrm{PI} 3 \mathrm{~K} / \mathrm{Akt}$ axis were investigated. 
We present the following article in accordance with the ARRIVE reporting checklist (available at http://dx.doi. org/10.21037/atm-20-8250).

\section{Methods}

\section{Animals and experimental design}

A total of 80 male Wistar rats (6 weeks old and weighing 200-300 g) were purchased from the Southern Model Animal Research Center (Nanjing, China). The animals were housed under a 12-hour-day light cycle with free access to food and water. The CHF model was induced by left anterior descending coronary artery ligation. The control group received the identical surgical procedure without artery ligation. At 4 weeks after the artery ligation operation, rats were divided into four groups: control group ( $N=20)$, CHF group ( $N=20)$, CHF rats with 8 weeks of aerobic exercise training [CHF-Tr, $(\mathrm{N}=20)]$, and CHFTr rats overexpressing MALAT1 [CHF-Tr-MALAT1, $(\mathrm{N}=20)]$. Over-expression of the lncRNA MALAT1 was performed via injection of $10 \mu \mathrm{L}$ pcDNA-MALAT 1 plasmid (Riobio, Guangzhou, China) at the commencement of aerobic exercise training according to the manufacturer's instructions. At 4 weeks post-surgery, several rats died and the final numbers in each group were as follows: $\mathrm{N}_{\text {Conrtol }}=19$ (1 died), $\mathrm{N}_{\mathrm{CHF}}=19$ (1 died), $\mathrm{N}_{\mathrm{CHF}-\mathrm{Tr}}=17$ (3 died), $\mathrm{N}_{\mathrm{CHF}^{-} \mathrm{Tr}-}$ MALAT1 $=18$ ( 2 died). The transfection efficiency of MALAT1 in the CHF rats was assessed via real-time polymerase chain reaction (RT-PCR). At the conclusion of the experiment, rats were anesthetized using $50 \mathrm{mg} / \mathrm{kg}$ pentobarbital sodium and the heart and lung tissues were harvested. Experiments were performed under a project license (2019-101) granted by the Ethics Committee of Beijing Xiaotangshan Hospital, in compliance with Beijing Xiaotangshan Hospital guidelines for the care and use of animals.

\section{Aerobic exercise training}

Four weeks after the left anterior descending coronary artery ligation, the CHF-Tr group and the CHF-TrMALAT1 group commenced aerobic exercise training by running on a treadmill over an 8 -week period. The training was conducted 5 times a week, with the treadmill speed set at $16 \mathrm{~m} / \mathrm{min}$. Initially, the exercise duration was 20 minutes for each session. After a 1-week adaptation, the weekly exercise time was extended by 10 minutes until each exercise duration reached 50 minutes (17).

\section{Determination of cardiac function}

At the conclusion of the experiment, the cardiac function was detected by echocardiography (ECG). The rats in each group were fixed in the left lateral position and ECG (probe frequency setting to $10 \mathrm{MHz}$ ) was performed according to the manufacturer's instructions. Maximal left ventricular pressure (dp/dtmax), left ventricular end-diastolic pressure (LVDEP), and left ventricular systolic pressure (LVSP) were recorded.

\section{Detection of inflammatory factors}

The left ventricular myocardial tissue was minced evenly and $1 \mathrm{~g}$ of the subsequent tissue was lysed in $2 \mathrm{~mL}$ RIPA protein lysis buffer. The levels of the serum inflammatory factors including tumor necrosis factor alpha (TNF- $\alpha$ ), interleukin-6 (IL-6), and interleukin-1 $\beta$ (IL-1 $\beta$ ) were assessed using enzyme-linked immunosorbent assay (ELISA) kits (Abcam, CA, USA) according to the manufacturer's instructions. The absorbance in each sample was detected with a microplate reader at $450 \mathrm{~nm}$ (Millipore, CA, USA).

\section{Masson staining}

Rats were anesthetized using $50 \mathrm{mg} / \mathrm{kg}$ pentobarbital sodium and the hearts were harvested and fixed in $4 \%$ formalin for 5 days. Paraffin sections of the left ventricular myocardial tissue were processed. After deparaffinization, the sections were hydrated using an ethanol gradient and hematoxylin and eosin (HE) staining was performed according to the manufacturer's instructions (Solarbio, Beijing, China). Finally, sections were dehydrated using an ethanol gradient, cleared with xylene, and sealed. Sections were observed under an optical microscope (Nikon, Tokyo, Japan) with $100 \times$ and $400 \times$ magnification.

\section{Immunohistochemical staining}

The paraffin sections of the left ventricular myocardial tissue were prepared as described above. After antigen retrieval with citrate buffer, $3 \%$ hydrogen peroxide was used to eliminate endogenous peroxidase activity. Sections were blocked with $0.05 \%$ bovine serum albumin (BSA; GIBCO, CA, USA) for 1 hour at room temperature, followed by incubation with primary antibodies (1:400 anti-collagen I antibody and 1:400 anti-collagen II antibody; Abcam, CA, USA) at $4{ }^{\circ} \mathrm{C}$ overnight. Sections were then incubated 
for 1 hour with a horseradish peroxidase (HRP)-labeled secondary antibody, followed by 3,3'-diaminobenzidine (DAB) staining (ZSbio, Beijing, China). A positive DAB stain can be visualized as a specific brown-yellow color. Finally, sections were dehydrated using an ethanol gradient, treated with xylene and sealed. Slides were observed under an optical microscope (Nikon, Tokyo, Japan) with 400× magnification.

\section{Immunofluorescence staining}

The paraffin sections of the left ventricular myocardial tissue were prepared as previously described. Sections were incubated in membrane permeation solution at $37^{\circ} \mathrm{C}$ for 10 minutes, followed by incubation with a blocking solution at $37^{\circ} \mathrm{C}$ for 10 minutes. Sections were then incubated with primary anti-light chain 3B antibodies (anti-LC3B-II; 1:400; Abcam, CA, USA) overnight at $4{ }^{\circ} \mathrm{C}$, followed by incubation with a fluorescent secondary antibody $(1: 200)$ at $37^{\circ} \mathrm{C}$ for 1 hour. The nucleus was stained with 4',6-diamidino-2phenylindole (DAPI; 1:1,000) for 10 minutes. Finally, slides were sealed with glycerol and observed under a fluorescence microscope (Nikon, Tokyo, Japan) with 400× magnification.

\section{Measurement of oxidative stress}

The left ventricular myocardial tissue was minced evenly, and $1 \mathrm{~g}$ was resuspended in $2 \mathrm{~mL}$ RIPA protein lysis buffer. The activity of superoxide dismutase (SOD), and the levels of malondialdehyde (MDA) and glutathione peroxidase (GSH-Px) were measured using ELISA kits (Solarbio, Beijing, China) according to the manufacturer's protocols. Finally, the absorbance in each sample was detected with a microplate reader (Millipore, CA, USA).

\section{Cell culture and intervention}

Primary rat cardiomyocytes were purchased from American Type Culture Collection (ATCC, Shanghai, China). To generate cells overexpressing the lncRNA MALAT1 (the MALAT1 group), cells were transfected with the MALAT1 plasmid for 8 hours using $5 \mu \mathrm{L}$ Lipofectamine 3000 (Invitrogen, LA, USA). Another group of cells were cotransfected with the MALAT1 plasmid and the miR-150-5p mimic (30 $\mu \mathrm{moL}$; Riobio, Guangzhou, China) to generate the MALAT1 + miR-150-5p group.

\section{Terminal deoxynucleotidyl transferase (TdT) dUTP Nick- End Labeling (TUNEL) apoptosis assay}

Myocardial apoptosis was detected via the TUNEL assay kit (Roche, Basel, Switzerland) according to the manufacturer's instructions. Cells were observed under a fluorescence microscope (Nikon, Tokyo, Japan) with 400× magnification. The blue fluorescence represents the nucleus stained by DAPI, and red fluorescence represents apoptotic cells stained by cyanine 3 .

\section{RT-PCR}

The total RNA was extracted from rat myocardial tissues using Trizol reagent (Invitrogen, LA, USA), and the RNA concentration was measured. The primer amplification was performed according to the manufacturer's protocols (Takara, Tokyo, Japan). The primers used were as follows: MALAT1 Forward: GAATTGCGTCATTTAAAGCCTAGTT; MALAT1 Reverse: GTTTCATCCTACCACTCCCAATTAAT; miR150-5p Forward: TATACGCTATCATAGTTTGCTG; miR-150-5p Reverse: GTTAGTGGTATTATGGTA; U6 Forward: GCTTCGGCAGCACATATACT; and U6 Reverse: AACGCTTCACGAATTTGCGT.

\section{Western blotting analysis}

Total protein was extracted from rat myocardial tissues using the RIPA reagent (Solarbio, Beijing, China) and the protein concentration was detected using the BCA method (Invitrogen, LA, USA). A 10\% gel was prepared for protein electrophoresis, and the proteins were then transferred onto a polyvinylidene difluoride (PVDF) membrane (Millipore, CA, USA). After blocking with $5 \%$ BSA at room temperature for 1 hour, the membrane was incubated with primary antibodies overnight at $4{ }^{\circ} \mathrm{C}$. The primary antibodies used were as follows: 1:400 LC3B (Abcam, CA, USA), 1:400 belin-1 (Cell Signaling Technology, LA, USA), 1:200 SQSTM1/p62 (sequestosome-1; Cell Signaling Technology, LA, USA), 1:800 GAPDH (glyceraldehyde 3-phosphate dehydrogenase; Santa Cruz, CA, USA), 1:800 PI3K/p-PI3K (phosphoinositide 3-kinase/ phosphorylated PI3K; Abcam, CA, USA), and 1:400 Akt/ p-Akt (protein kinase B/phosphorylated-Akt; Cell Signaling Technology, LA, USA). Membranes were then incubated with the secondary antibody $(1: 1,000)$ for 1 hour at room 
temperature. After adding the enhanced chemiluminescence (ECL) solution (Solarbio, Beijing, China), the protein bands were scanned with the Odyssey scanner (Invitrogen, CA, USA).

\section{Statistical analysis}

GraphPad Prism 7.0 was used for data analysis. All data are shown as the mean \pm standard deviation (SD). Unpaired student's $t$-test was used to detect significant differences between two groups. A difference with a $\mathrm{P}$ value $<0.05$ was considered statistically significant.

\section{Results}

\section{Aerobic exercise improved cardiac function indexes in rats with $\mathrm{CHF}$}

To determine the transfection efficiency of the lncRNA MALAT1 plasmid, RT-PCR was performed to detect the levels of serum MALAT1 in the CHF-Tr group and the CHF-Tr-MALAT1 group. As shown in Figure 1A, the expression of MALAT1 was significantly higher in the CHF-Tr-MALAT1 group compared to the CHF-Tr group $(\mathrm{P}<0.01)$. Compared to the control group, rats with $\mathrm{CHF}$ showed evidently lower dp/dtmax and LVSP, while heart rate and LVDEP were evidently higher (Figure 1B,C,D,E). The trends for these indexes were reversed in CHF rats that underwent aerobic exercise, indicating that aerobic exercise improved cardiac function indexes in rats with $\mathrm{CHF}$ (Figure 1B,C,D,E). Interestingly, all beneficial effects of aerobic exercise on CHF rats were eliminated by the overexpression of MALAT1. In particular, overexpression of MALAT1 resulted in deterioration of the cardiac function indexes in CHF rats, including higher heart rate and LVDEP, and lower dp/dtmax and LVSP (Figure 1B,C,D,E).

\section{Aerobic exercise inbibited left ventricular remodeling in rats with $\mathrm{CHF}$}

The ratio of left ventricle weight to body weight reflects the degree of left ventricular hypertrophy, and the ratio of lung weight to body weight presents the degree of pulmonary edema which is a common complication of left heart failure. Rats with CHF displayed greater heart compared to control rats (Figure $2 A$ ), especially the left ventricular weight (Figure 2B). Additionally, the lung weights of CHF rats were larger than control rats (Figure 2C). An obvious decrease in the lung and left ventricular weights were observed in $\mathrm{CHF}$ rats receiving aerobic exercise (Figure $2 A, B, C$ ). The Masson's trichrome staining demonstrated that fibrosis of the myocardial tissue (stained blue) was ameliorated by aerobic exercise in CHF rats (Figure 2D). Moreover, the levels of collagen I and collagen II expression were also markedly reduced following aerobic exercise in $\mathrm{CHF}$ rats, as demonstrated by immunohistochemical staining (Figure 2E,F). The mRNA levels of collagen I and collagen II confirmed these results (Figure 2G). Overexpression of MALAT1 halted the anti-fibrotic effects of aerobic exercise in CHF rats (Figure $2 A, B, C, D, E, F, G$ ), indicating that MALAT1 may be a target in aerobic exercise during CHF treatment.

\section{Aerobic exercise reduced oxidative stress and inflammation in rats with $\mathrm{CHF}$}

ROS and inflammatory markers play an important role in the pathophysiology of CHF $(18,19)$. The MMPs are responsible for the degradation of the myocardial extracellular matrix (ECM), and imbalance of the ECM may lead to systolic and diastolic dysfunction of the heart, resulting in CHF (20). The levels of SOD and GSH$\mathrm{Px}$ expression were significantly down-regulated in $\mathrm{CHF}$ rats, while the MDA levels were obviously up-regulated (Figure $3 A, B, C$ ). Moreover, levels of the inflammatory cytokines including TNF- $\alpha$, IL- 6 , and IL- $1 \beta$ were significantly higher in $\mathrm{CHF}$ rats compared to control rats. The inflammatory mediums containing MMP-2 and MMP9 were also increased in CHF rats (Figure 3D,E, F, G,H). Aerobic exercise markedly decreased all these inflammatory cytokines and inflammatory mediums, and overexpression of MALAT1 ameliorated the effects of aerobic exercise (Figure $3 A, B, C, D, E, F, G, H)$. These results demonstrated that aerobic exercise reduced the oxidative stress and inflammation in CHF rats, and the mechanism was potentially mediated through MALAT1.

\section{Aerobic exercise decreased apoptosis and increased autophagy in rats with $\mathrm{CHF}$}

According to data from the TUNEL assays, myocardial apoptosis in CHF rats was markedly increased compared to control rats (Figure 4A,B). Aerobic exercise reduced the number of apoptotic cells in the myocardial tissue of CHF rats, while overexpression of MALAT1 reduced the anti- 
A

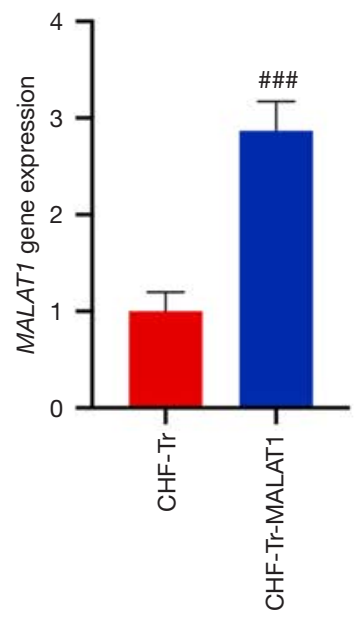

$\mathrm{D}$

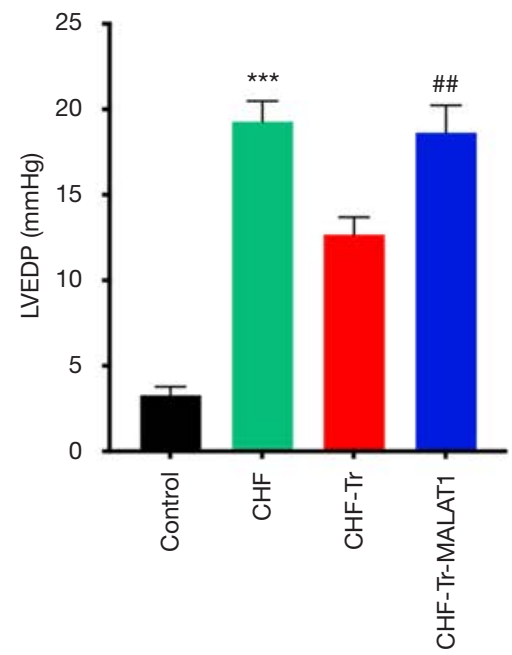

B

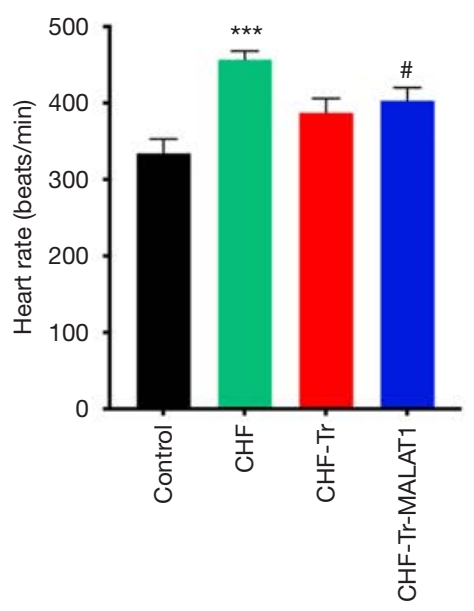

C

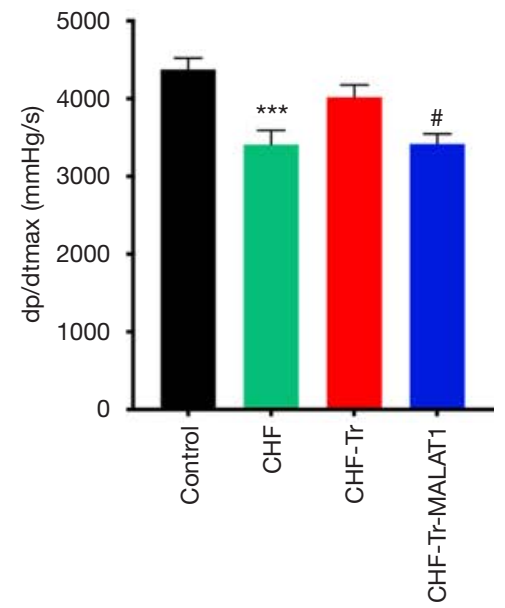

$\mathrm{E}$

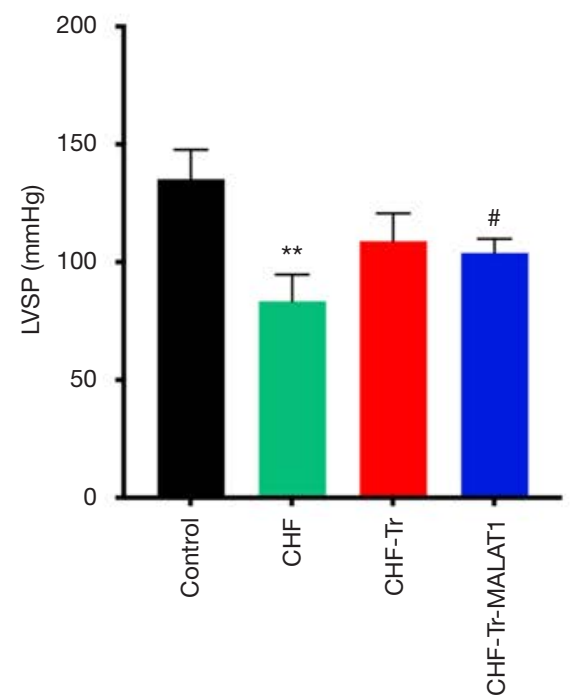

Figure 1 Aerobic exercise improved the cardiac function indexes in rats with chronic heart failure. The CHF rat model was induced via left anterior descending coronary artery ligation. Four weeks after the surgery, CHF rats received aerobic exercise training over an 8-week period (the CHF-Tr group). CHF-Tr rats were injected with the pcDNA-MALAT1 plasmid (the CHF-Tr-MALAT1 group). (A) The expression of the lncRNA MALAT1 in myocardial tissues was measured by RT-PCR. After 8 weeks, ECG was performed to assess the left ventricular function indexes including (B) the heart rate; (C) dp/dtmax; (D) LVDEP; and (E) LVSP. $N_{\text {onrtol }}=19, N_{C H F}=19, N_{C H F-T r}=17$, $\mathrm{N}_{\text {CHF-Tr-MALAT1 }}=18$. Data are shown as mean $\pm \mathrm{SD}$. Statistically significant differences compared to the control group are shown as ${ }^{* *}, \mathrm{P}<0.01$; and ${ }^{* * *}, \mathrm{P}<0.001$; and compared to the CHF-Tr group as ${ }^{*}, \mathrm{P}<0.05$; ${ }^{\# \#}, \mathrm{P}<0.01$; and ${ }^{\# \# \#,} \mathrm{P}<0.001$. CHF, chronic heart failure; MALAT1, metastasis-associated lung adenocarcinoma transcript 1; ECG, echocardiography; dp/dtmax, maximal left ventricular pressure rising rate; LVDEP, left ventricular end-diastolic pressure; LVSP, left ventricular systolic pressure; SD, standard deviation. 
A
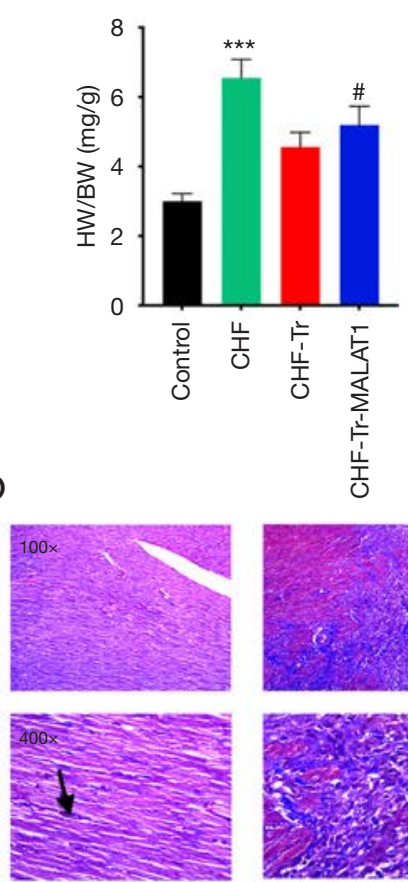

Control

$\mathrm{E}$

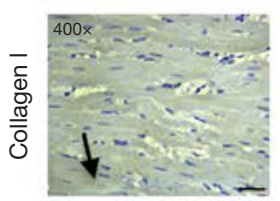

Control

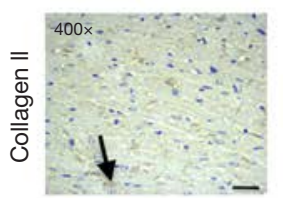

Control
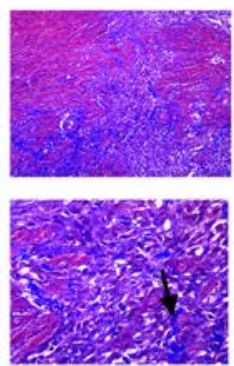

CHF

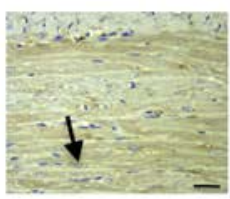

$\mathrm{CHF}$

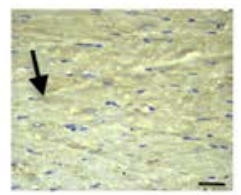

$\mathrm{CHF}$
B
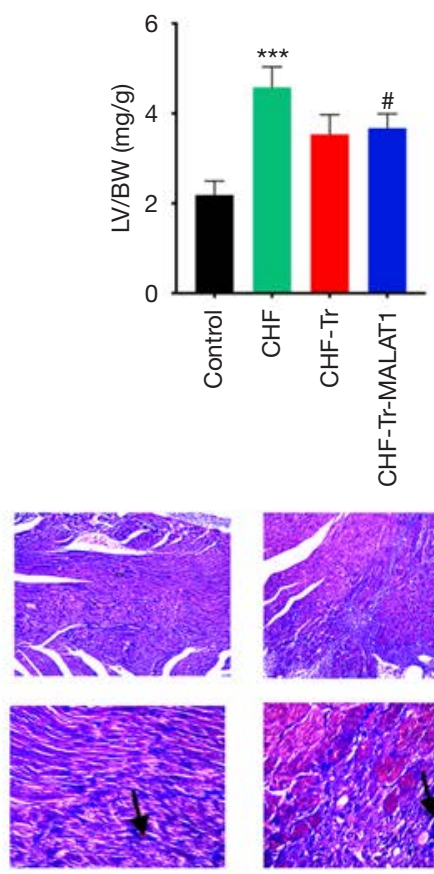

CHF-Tr

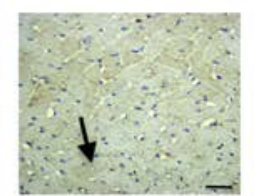

CHF-Tr

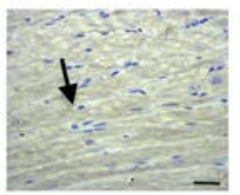

CHF-Tr
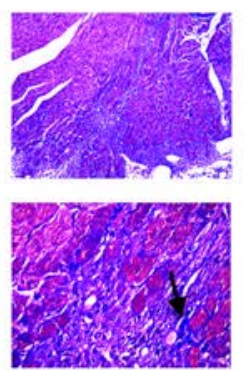

CHF-Tr-MALAT1

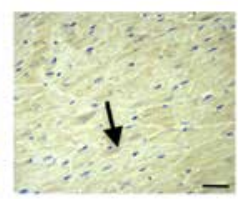

CHF-Tr-MALAT1

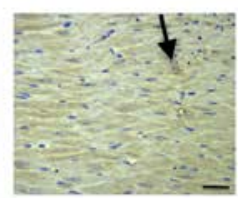

CHF-Tr-MALAT1
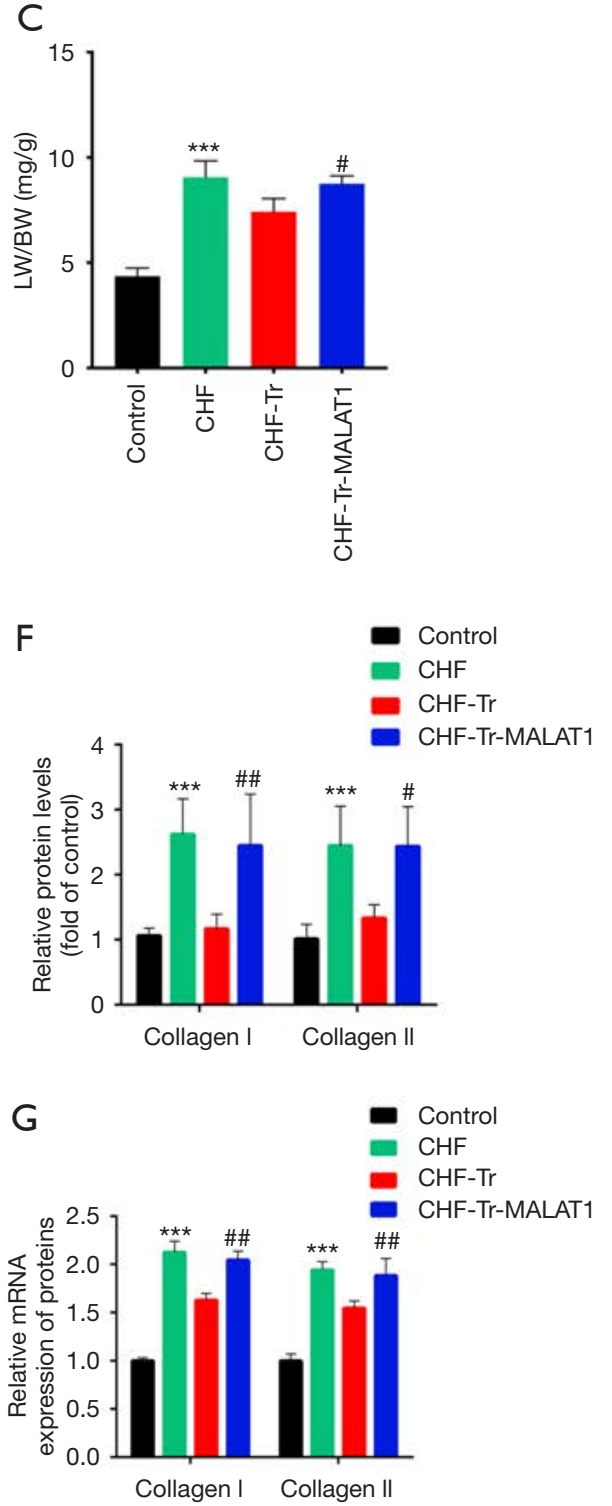

Figure 2 Aerobic exercise inhibited left ventricular remodeling in rats with chronic heart failure. The CHF rat model was induced via left anterior descending coronary artery ligation. Four weeks after the surgery, CHF rats receiving aerobic exercise training over an 8 -week period were designated the CHF-Tr group. CHF-Tr rats that were injected with the pcDNA-MALAT1 plasmid were designated the CHFTr-MALAT1 group. (A) The ratio of heart weight to body weight was measured. (B) The ratio of left ventricle weight to body weight was measured. (C) The ratio of lung weight to body weight was measured. (D) Masson staining was performed on rat left ventricular tissue to assess the degree of left ventricular fibrosis in each group. The fibrous tissue was stained blue and was shown by the black arrow in the figure. 100x magnification, scale bar $=100 \mu \mathrm{m} ; 400 \times$ magnification, scale bar $=20 \mu \mathrm{m}$. (E) The immunohistochemical staining of collagen I and collagen II was performed, and the positive staining was and was shown by the black arrow in the figure. $400 \times$ magnification, scale bar $=20 \mu \mathrm{m}$. (F) The statistical analysis of immunohistochemical staining to assess the content of collagen in each group. (G) The mRNA expressions of collagen I and collagen II in each group as measured by RT-PCR. $\mathrm{N}_{\text {Conrtol }}=19, \mathrm{~N}_{\mathrm{CHF}}=19, \mathrm{~N}_{\mathrm{CHF}-\mathrm{Tr}}=17, \mathrm{NCHF}-\mathrm{Tr}-\mathrm{MALAT} 1=18$. Data are shown as mean $\pm \mathrm{SD}$. Statistically significant differences compared to the control group are shown as ***, $\mathrm{P}<0.001$; and compared to the CHF-Tr group as ", $\mathrm{P}<0.05 ;{ }^{* \#}, \mathrm{P}<0.01$. HW, heart weight; BW, body weight; LV, left ventricle; LW, lung weight; CHF, chronic heart failure; MALAT1, metastasis-associated lung adenocarcinoma transcript 1 ; SD, standard deviation. 
A
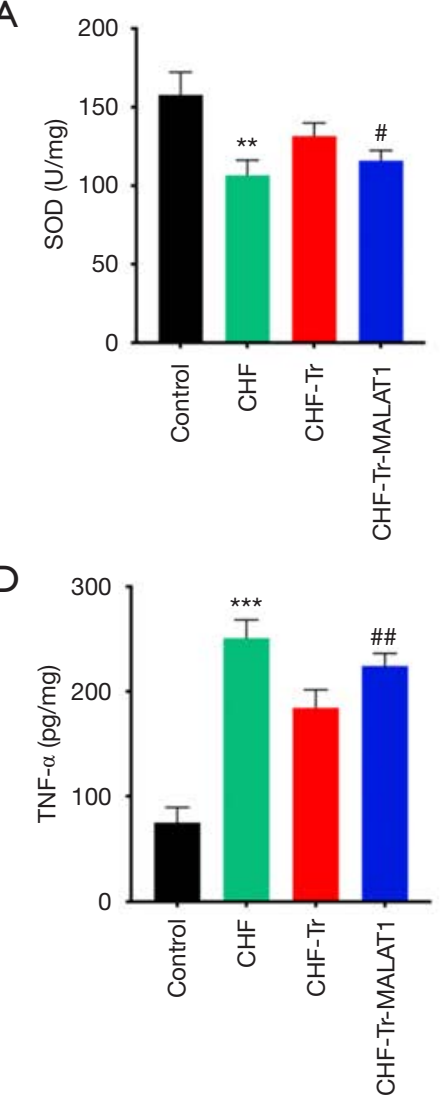

G

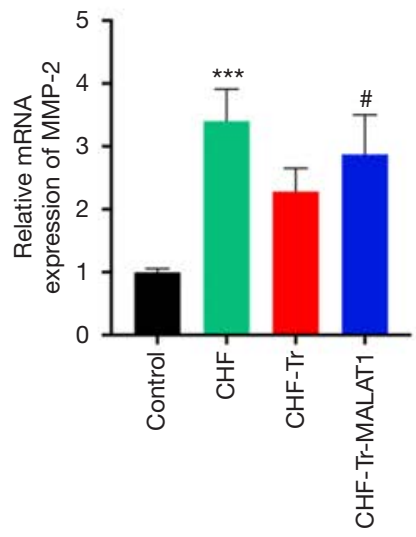

B

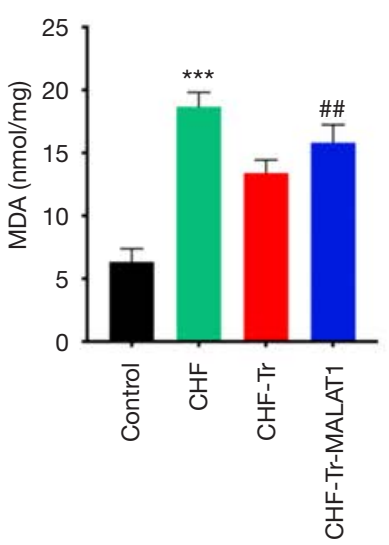

E

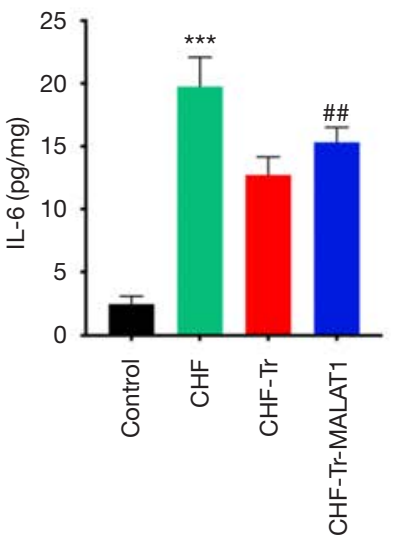

$\mathrm{H}$

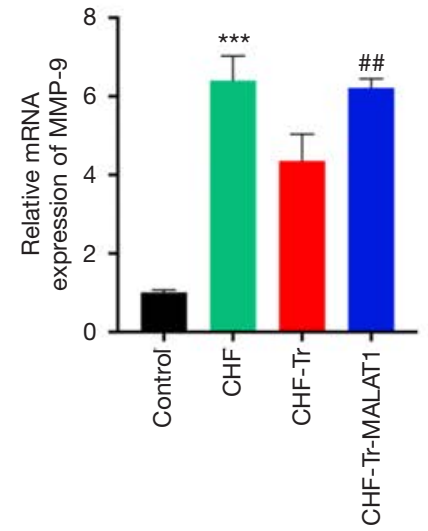

C

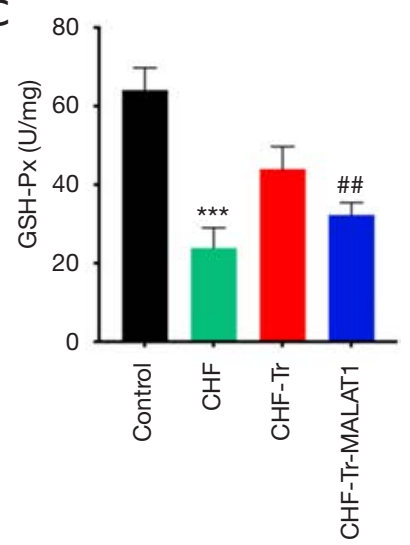

$\mathrm{F}$

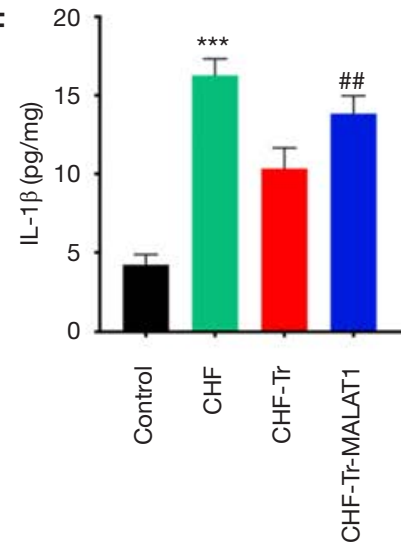

Figure 3 Aerobic exercise reduced oxidative stress and inflammation in rats with chronic heart failure. The CHF rat model was induced via left anterior descending coronary artery ligation. Four weeks after the surgery, CHF rats receiving aerobic exercise training over an 8-week period were designated the CHF-Tr group. CHF-Tr rats injected with pcDNA-MALAT1 plasmid were designated the CHF-Tr-MALAT1 group. (A) The activity of SOD was detected by the xanthine oxidase method. (B) The determination of MDA content was performed by the thiobarbituric acid staining method. (C) Levels of GSH-Px were detected by colorimetric methods with the GSH-Px assay kit. ELISA kits were used to measure the levels of the inflammatory cytokines including (D) TNF- $\alpha$; (E) IL-6; and (F) IL-1 $\beta$. RT-PCR was used to assess the mRNA expressions of the inflammatory mediums containing (G) MMP-2; and (H) MMP-9. $\mathrm{N}_{\text {Conrtol }}=19, \mathrm{~N}_{\mathrm{CHF}}=19, \mathrm{~N}_{\mathrm{CHF}-\mathrm{Tr}}=17$, $\mathrm{N}_{\text {CHF-Tr-MALAT1 }}=18$. Data are shown as mean $\pm \mathrm{SD}$. Statistically significant differences compared to the control group are shown as $* *, P<0.01$; and ${ }^{* * *}, \mathrm{P}<0.001$; and compared to the CHF-Tr group as ${ }^{\#}, \mathrm{P}<0.05$; $\#, \mathrm{P}<0.01$. CHF, chronic heart failure; MALAT1, metastasis-associated lung adenocarcinoma transcript 1; SOD, superoxide dismutase; MDA, malondialdehyde; GSH-Px, glutathione peroxidase; TNF- $\alpha$, tumor

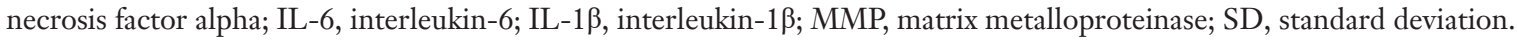


apoptotic effects of aerobic exercise. This indicated that aerobic exercise protected myocardial cells from injury via inhibiting MALAT1. Autophagy, also known as type II programmed cell death, is involved in the occurrence and development of many diseases. A proper degree of autophagy has a protective effect on cells (21). As shown in Figure $4 C$, expression of the autophagy-related proteins LC3B-II and beclin-1 were down-regulated in CHF rats. The fluorescent staining of LC3B-II showed a similar trend in each group (Figure 4D,E). Moreover, the protein expression of the marker of autophagic flux SQSTM1/p62 increased. These results demonstrated that autophagy was suppressed in CHF rats (Figure 4D). Aerobic exercise in $\mathrm{CHF}$ rats restored the autophagy, and overexpression of MALAT1 again suppressed the autophagy (Figure 4C,D,E).

\section{Aerobic exercise regulated the MALAT1/miR-150-5p/ PI3K/Akt signaling patbway in rats with CHF}

Previous studies have reported that MALAT1 could play a role in various diseases by interacting with miRNAs (22). To investigate the potential downstream mechanisms of MALAT1 in CHF, miRcode was used to search for target miRNAs related to MALAT1. After predicting the potential miRNA binding sites in MALAT1, miR-150-5p was identified as a potential target of MALAT1. Figure $5 \mathrm{~A}$ shows that the levels of MALAT1 in CHF rat myocardial tissue was significantly up-regulated, and aerobic exercise markedly inhibited its expression. The levels of miR-150-5p expression were inversely related to MALAT1 expression in each rat treatment group (Figure 5B). The PI3K/Akt signaling pathway has been reported to play an important role in CHF (23). Western blot analysis showed that the protein expressions of $\mathrm{p}-\mathrm{PI} 3 \mathrm{~K}$ and $\mathrm{p}$-Akt increased in $\mathrm{CHF}$ rats, and this was inhibited by aerobic exercise (Figure 5C). Restoring the aerobic exercise-suppressed MALAT1 levels reduced the expression of $\mathrm{p}-\mathrm{PI} 3 \mathrm{~K}$ and $\mathrm{p}-\mathrm{Akt}$. These data indicated that the effects of aerobic exercise on CHF were mediated by the interaction between MALAT1 and miR$150-5 \mathrm{p}$, and inactivation of the downstream PI3K/Akt signaling pathway.

\section{Over-expression of MALAT1 regulated the miR- 150-5p/PI3K/Akt signaling pathway in primary rat cardiomyocytes}

The mechanisms of aerobic exercise in CHF were further investigated in vitro. Primary rat cardiomyocytes were transfected with the MALAT1 plasmid to overexpress MALAT1 (Figure 6A). Overexpression of MALAT1 markedly decreased the levels of miR-150-5p (Figure $6 B$ ). The protein expression of p-PI3K and p-Akt were significantly increased, indicating activation of PI3K/Akt signaling pathway (Figure 6C). Furthermore, overexpression of MALAT1 resulted in greater cellular apoptosis (Figure 6D,E) and less autophagy (Figure 6F,G,H). Primary rat cardiomyocytes were further co-transfected with the MALAT1 plasmid and the miR-150-5p mimic. The miR-150-5p levels were restored in the MALAT1+miR-150$5 \mathrm{p}$ group (Figure $6 B$ ) and the PI3K/Akt signaling pathway was suppressed (Figure 6C). When the levels of miR-150-5p expression were restored, a reduction of cellular apoptosis was observed and the levels of autophagy were subsequently increased (Figure 6F,G,H). The above results further verified the data from the in vivo experiments. However, a dualluciferase reporter assay is needed to confirm that miR-150$5 \mathrm{p}$ is a direct target of MALAT1.

\section{Discussion}

LncRNAs are the most prominent transcriptomes in the heart but its role has not yet been fully elucidated. In the past decade, MALAT1 was reported to be related closely with cancer metastasis. Knockdown of MALAT1 inhibited the proliferation, migration and Invasion in non-smallcell lung cancer cells via modulating the miR-515-3p/ TRIM65 axis (24). In thyroid cancer (TC) progression, long noncoding RNA MALAT1 had stimulatory effect on proliferation, migration, and invasion of TC cells (25). Recently, MALAT1 has been reported to be expressed abundantly in myocardial tissues (26). In the type 1 diabetes mellitus (T1DM) streptozotocin rat model, the levels of MALAT1 in myocardial tissues was significantly increased (27). Knock-down of MALAT1 with the small interfering RNA in T1DM rats significantly improved the inflammation and myocardial apoptosis (27). The expression of the MALAT1 gene in myocardial tissue of rats receiving intermittent hypoxia with hypercapnia (IHH) for 3 weeks was significantly higher than that in the control group. Increased levels of hypoxia-induced inflammatory factors including hypoxia inducible factor$1 \alpha$ (HIF-1 $\alpha)$, toll-like receptor 4 (TLR4), and IL-6 were also observed, indicating that MALAT1 might regulate obstructive sleep apnea (OSA)-induced myocardial immune injury (28). Moreover, in women with pregnancy-induced hypertension (PIH), levels of serum MALAT1 was up- 

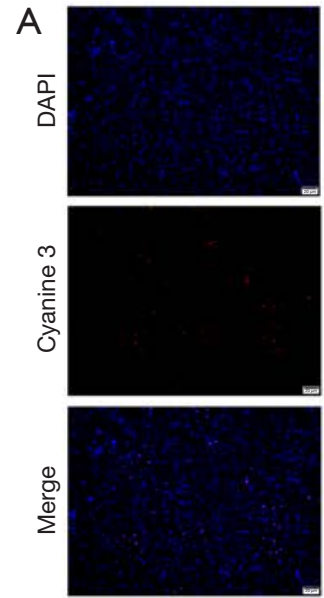

Control

D
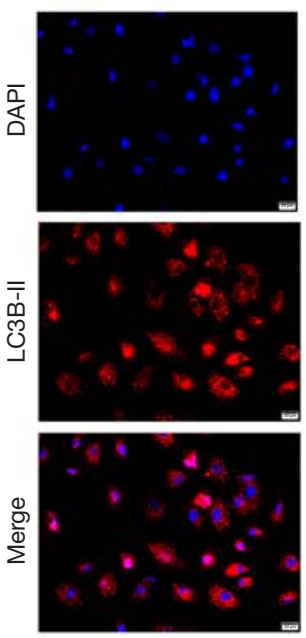

Control
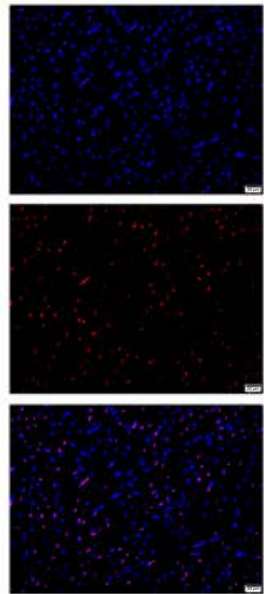

$\mathrm{CHF}$
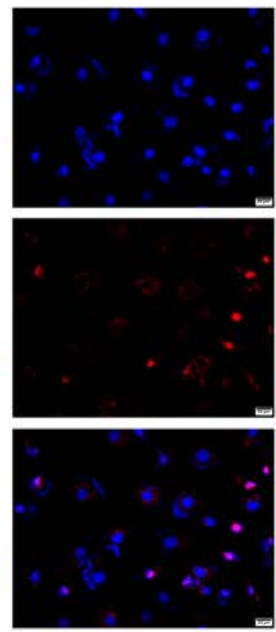

CHF
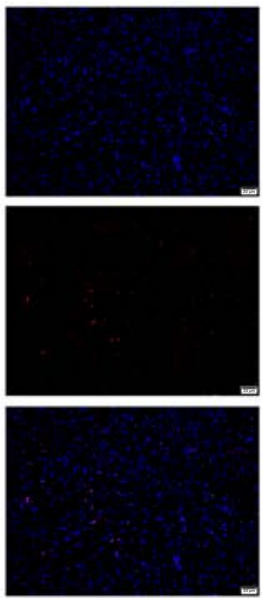

CHF-Tr
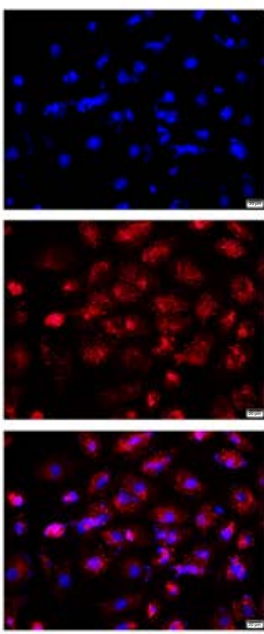

CHF-Tr
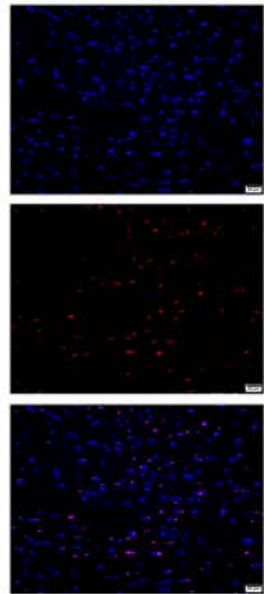

CHF-Tr-MALAT1
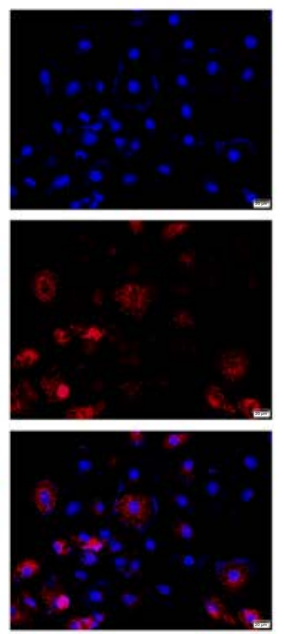

CHF-Tr-MALAT1
B

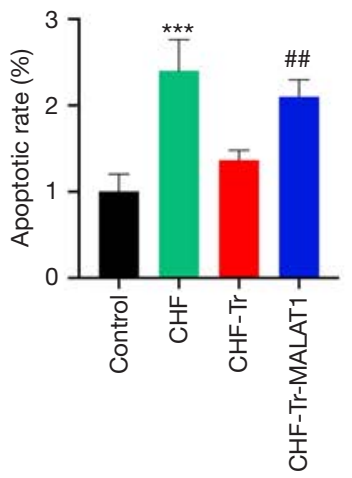

C

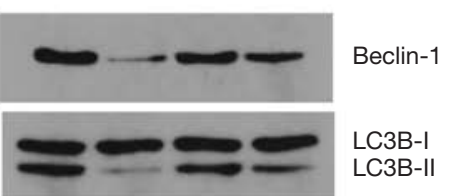

$\mathrm{E}$

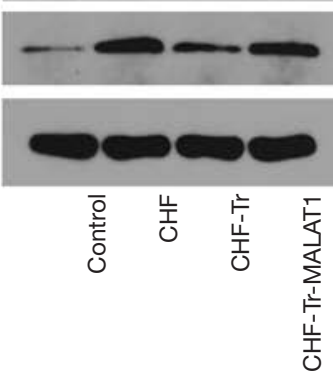

SQSTM1/p62

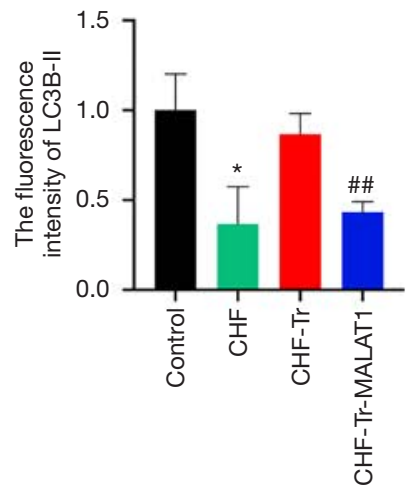

Figure 4 Aerobic exercise decreased apoptosis and increased autophagy in rats with chronic heart failure. The CHF rat model was induced via left anterior descending coronary artery ligation. Four weeks after the surgery, CHF rats receiving aerobic exercise training over an 8-week period were designated the CHF-Tr group. CHF-Tr rats injected with the pcDNA-MALAT1 plasmid were designated the CHF-Tr-MALAT1 group. (A) The detection of cellular apoptosis in rat left ventricular tissue was performed by the TUNEL assay. The blue fluorescence represents the nucleus stained by DAPI, and red fluorescence represents apoptotic cells stained by cyanine 3. 400x magnification, scale bar $=20 \mu \mathrm{m}$. (B) Statistical analysis of the TUNEL assay was performed to assess the apoptotic rate in each group. (C) Western blot analysis was performed to detect autophagy-related proteins. (D) The immunofluorescence staining of LC3B-II was observed by fluorescence microscope. The blue fluorescence represents the nucleus stained by DAPI, and red fluorescence represents LC3B-II protein in the cytoplasm. 400x magnification, scale bar $=20 \mu \mathrm{m}$. (E) The statistical analysis of fluorescence intensity of LC3B-II in each group. $\mathrm{N}_{\text {Conrtol }}=19, \mathrm{~N}_{\mathrm{CHF}}=19, \mathrm{~N}_{\mathrm{CHF}-\mathrm{Tr}}=17, \mathrm{~N}_{\mathrm{CHF}-\mathrm{TT}-\mathrm{MALAT1}}=18$. Data are shown as mean \pm SD. Statistically significant differences compared to the control group are shown as *, $\mathrm{P}<0.05$; and ${ }^{* * *}, \mathrm{P}<0.001$; and compared to the CHF-Tr group as ${ }^{\# \#,} \mathrm{P}<0.01$. CHF, chronic heart failure; MALAT1, metastasis-associated lung adenocarcinoma transcript 1; LC3B, light chain 3B; SD, standard deviation. 
A

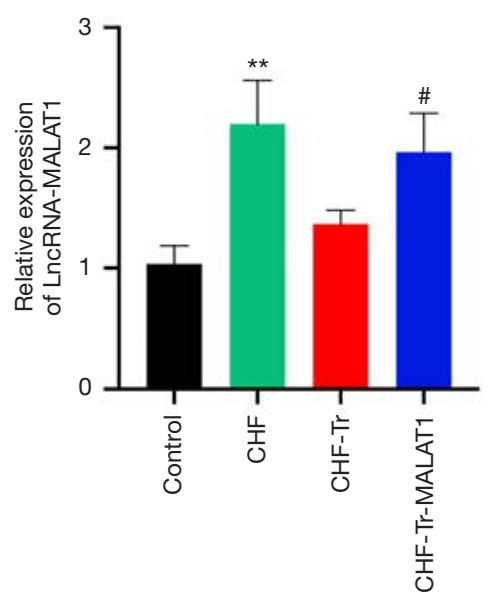

B

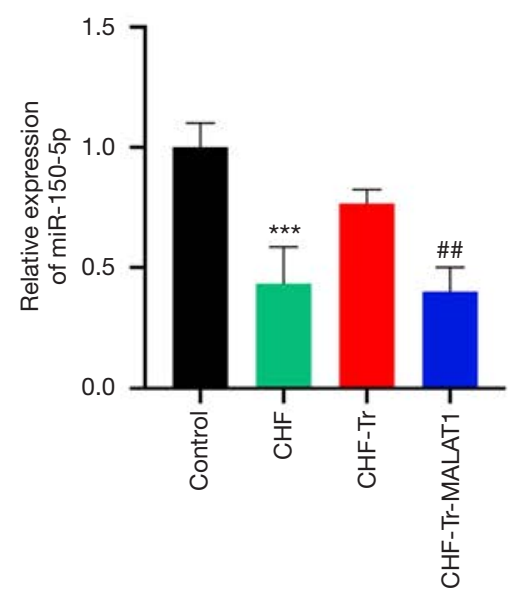

C

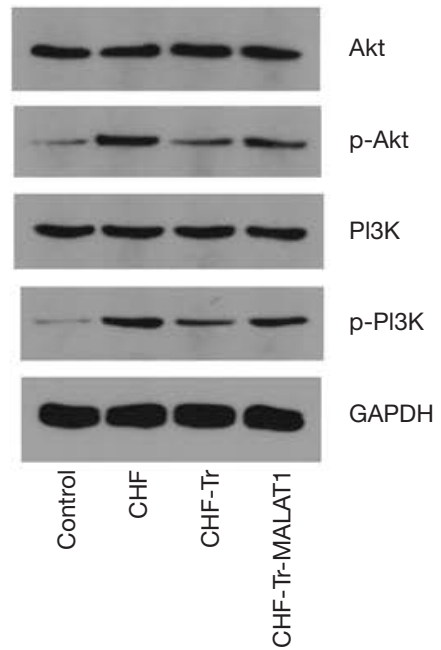

Figure 5 Aerobic exercise regulated the MALAT1/miR-150-5p/PI3K/Akt signaling pathway in rats with chronic heart failure. The $\mathrm{CHF}$ rat model was induced via left anterior descending coronary artery ligation. Four weeks after the surgery, CHF rats receiving aerobic exercise training over an 8-week period were designated the CHF-Tr group. CHF-Tr rats injected with the pcDNA-MALAT1 plasmid were designated the CHF-Tr-MALAT1 group. (A) The expression of lncRNA MALAT1 in each group was measured by RTPCR. (B) The expression of miR-150-5p in each group was measured by RT-PCR. (C) The protein levels of the PI3K/Akt signaling pathway were measured by western blot analysis. $\mathrm{N}_{\text {Conrtol }}=19, \mathrm{~N}_{\mathrm{CHF}}=19, \mathrm{~N}_{\mathrm{CHF}-\mathrm{Tr}}=17, \mathrm{~N}_{\mathrm{CHF}-\mathrm{Tr}-\mathrm{MALAT1}}=18$. Data are shown as mean \pm SD. Statistically significant differences compared to the control group are shown as ${ }^{* *}, \mathrm{P}<0.01$, and ***, $\mathrm{P}<0.001$; and compared to the $\mathrm{CHF}$ Tr group as \#, $\mathrm{P}<0.05 ;$ \#, $\mathrm{P}<0.01$. CHF, chronic heart failure; MALAT1, metastasis-associated lung adenocarcinoma transcript 1 ; PI3K, phosphatidylinositol 3 kinase; p-PI3K, phosphorylated phosphatidylinositol 3 kinase; Akt, protein kinase B; p-Akt, phosphorylated protein kinase B; RT-PCR, reverse transcription polymerase chain reaction; SD, standard deviation.

regulated while miR-150-5p was down-regulated (29). The activation of oxidative stress and inflammation contributed to the development of $\mathrm{PIH}$, potentially through regulating the miR-150-5p/endothelin-1 axis. All these studies demonstrated that MALAT1 plays an important role in cardiovascular-related diseases, and has a regulatory effect on inflammation and oxidative stress-related pathological mechanisms. However, there is currently insufficient research on the role of MALAT1 in cardiovascular diseases, especially in heart failure. This current report demonstrated that the expression of MALAT1 was significantly increased in $\mathrm{CHF}$ rats, which coincided with marked inflammation and oxidative stress. Aerobic exercise reduced the levels of MALAT1 and increased the levels of miR-150-5p. Considering the aerobic exercise-induced inhibition of inflammation and oxidative stress in CHF rats, MALAT1 might be crucial in the aerobic exercise treatment of CHF. This study suggested a novel concept that MALAT1 may be related to inflammation and oxidative stress in CHF, and this may contribute to improved understanding of the therapeutic mechanisms of aerobic exercise in CHF.

This investigation also found that the PI3K/Akt signaling pathway was activated by aerobic exercise in CHF, and it was suppressed by over-expression of MALAT1 (Figure 5C). Further in vitro experiments indicated that the potential downstream mechanisms of MALAT1 in aerobic exercise-treated $\mathrm{CHF}$ was mediated through regulation of the miR-150-5p/ PI3K/Akt axis. The relevance between miR-150-5p and PI3K/Akt signaling in CHF has not been previously reported. In T-cell acute lymphoblastic leukemia (T-ALL), miR-150 regulated cell growth and apoptosis via the PI3K/Akt pathway (30). Another study in natural killer (NK)/T cell lymphoma reported that miR-150 improved radiosensitivity by suppressing the $\mathrm{PI} 3 \mathrm{~K} / \mathrm{Akt} / \mathrm{mammalian}$ target of rapamycin (mTOR) pathway (31). In malignant lymphoma, overexpression of $\mathrm{miR}-150$ in NK/T-cell lymphoma cells increased the apoptotic rate and reduced telomerase activity through activation of the PI3K/Akt 
Page 12 of 16

A

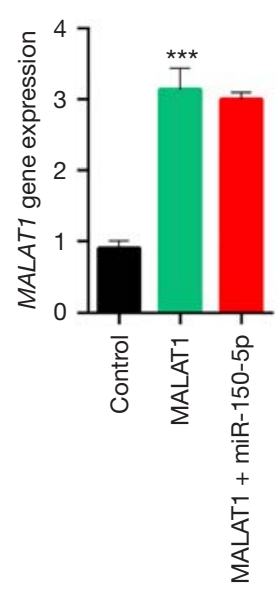

D
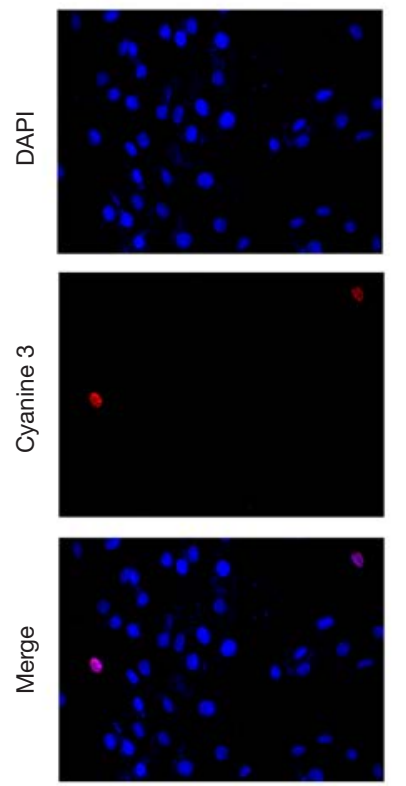

Control
B

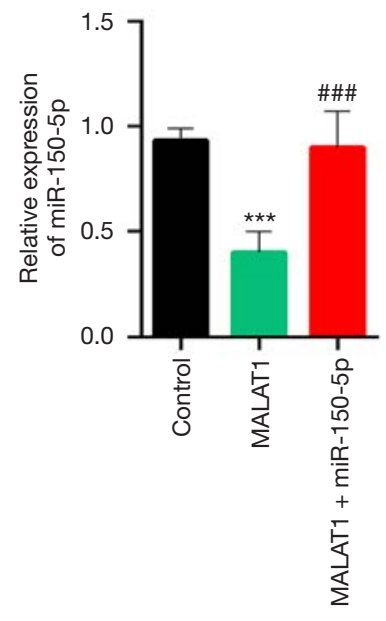

Hu et al. Aerobic exercise inhibits MALAT1 to improve CHF

C

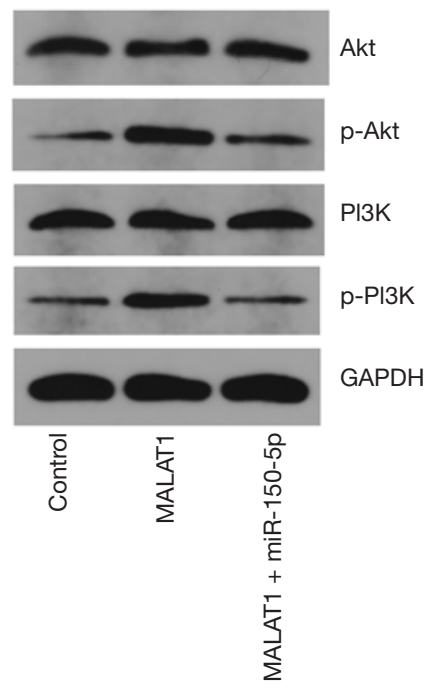

E

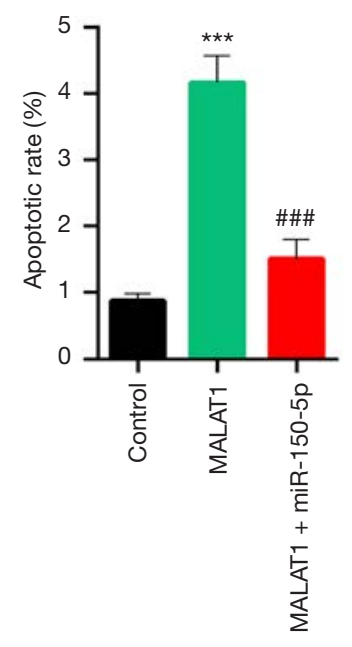

F
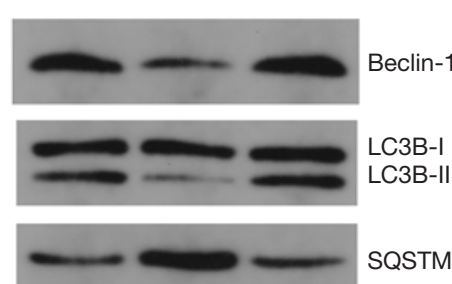
SQSTM1/p62

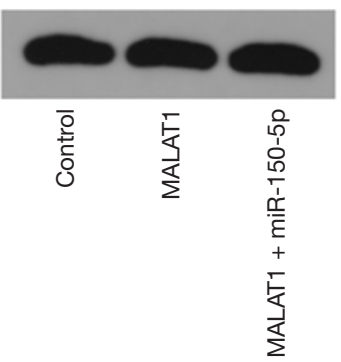
GAPDH 
G
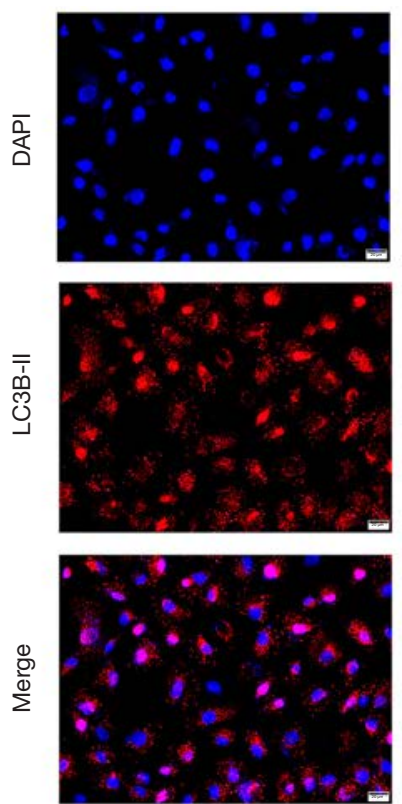

Control
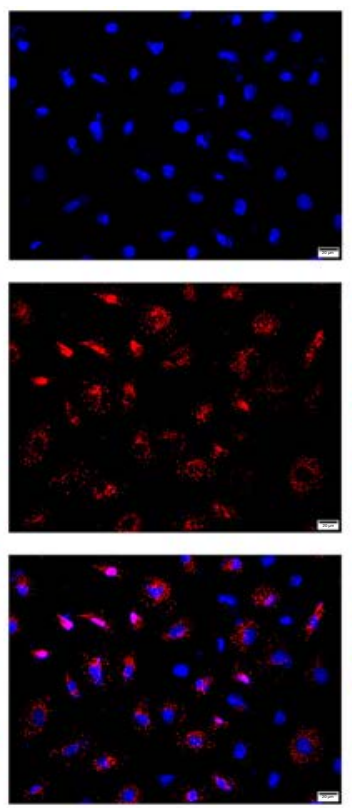

MALAT1
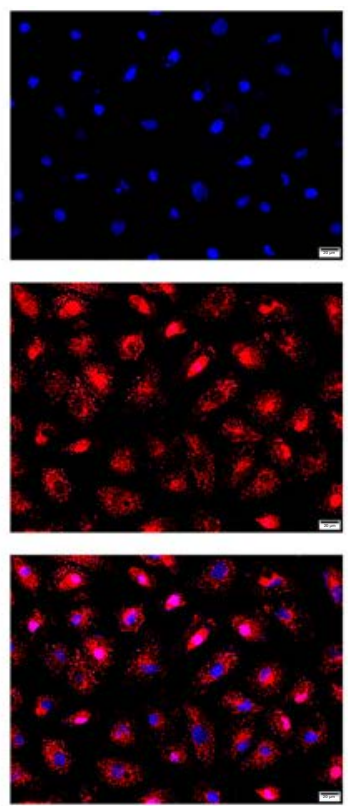

MALAT1 + miR-150-5p
$\mathrm{H}$

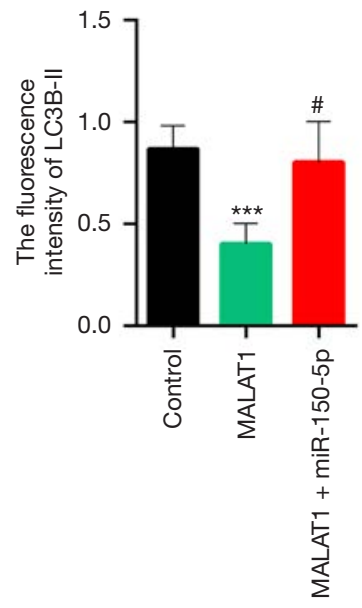

Figure 6 Over-expression of lncRNA MALAT1 regulated the miR-150-5p/PI3K/Akt signaling pathway in primary rat cardiomyocytes. The primary rat cardiomyocytes transfected with lncRNA MALAT1 plasmid to over-express lncRNA MALAT1 were designated the MALAT1 group. Cells co-transfected with the MALAT1 plasmid and the miR-150-5p mimic were designated the MALAT1+miR-150-5p group. (A) The expression of MALAT1 in each group was measured by RT-PCR. (B) The expression of miR-150-5p in each group was measured by RT-PCR. (C) The protein levels of the PI3K/Akt signaling pathway were measured by western blot analysis. (D) The detection of cellular apoptosis was performed by the TUNEL assay. The blue fluorescence represents the nucleus stained by DAPI, and red fluorescence represents apoptotic cells stained by cyanine 3. 400x magnification, scale bar $=20 \mu \mathrm{m}$. (E) Statistical analysis of the TUNEL assay was performed to assess the apoptotic rate in each group. (F) The detection of autophagy-related proteins was measured by western blot analysis. (G) The immunofluorescence staining of LC3B-II was observed by fluorescence microscope. The blue fluorescence represents the nucleus stained by DAPI, and red fluorescence represents the LC3B-II protein in the cytoplasm. 400x magnification, scale bar $=20 \mu \mathrm{m}$. (H) The statistical analysis of fluorescence intensity of LC3B-II in each group. Each experiment was repeated three times independently. Data are shown as mean $\pm \mathrm{SD}$. Statistically significant differences compared to the control group are shown as ${ }^{* * *}, \mathrm{P}<0.001$; and compared to the

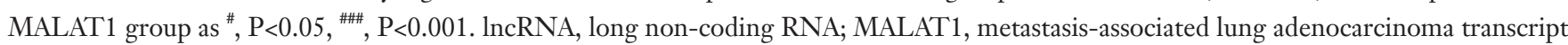
1; PI3K, phosphatidylinositol 3 kinase; p-PI3K, phosphorylated phosphatidylinositol 3 kinase; Akt, protein kinase B; p-Akt, phosphorylated protein kinase B; LC3B, light chain 3B; GAPDH, glyceraldehyde 3-phosphate dehydrogenase; RT-PCR, reverse transcription polymerase chain reaction; TUNEL, terminal deoxynucleotidyl transferase dUTP nick end labeling; DAPI, 4',6-diamidino-2-phenylindole; SD, standard deviation.

pathway (32). Crosstalk between miR-150-5p and the PI3K/ Akt axis has been confirmed in immune cells (32). However, this current study only demonstrated that the PI3K/Akt pathway is indeed regulated by MALAT1/miR-150-5p, but whether other signaling molecules are involved remains to be verified. Further experiments using the dual luciferase reporter assay will be necessary to assess the direct binding of miR-150-5p and the PI3K/Akt axis.
Aerobic exercise has been used for the diagnosis, treatment, and prognosis of CHF (33). More specifically, the interaction between aerobic exercise and $\mathrm{CHF}$ can be divided into two important stages: before the onset of $\mathrm{CHF}$ and CHF. For the primary prevention of CHF, a population-based prospective study found that men with long-term fitness levels had markedly lower risk of developing CHF (34). Florido et al. (35) reported that 
exercise was negatively associated with subclinical chronic myocardial damage, indicating that exercise reduced the risk of CHF. A prospective cohort study of 370,000 subjects found that there was a dose-dependent linear association between exercise and the risk of heart failure (36). Therefore, increased exercise can prevent CHF. For the secondary prevention of $\mathrm{CHF}$, aerobic exercise has been proven to be an effective treatment for CHF patients (37). A clinical study found that gradually increasing exercise volume can gradually lead to increased cardiac output. The arteriovenous oxygen content difference was significantly decreased after exercise, which suggested that the blood flow had redistributed after exercise (38). Clinical studies have found that different forms of exercise including continuous moderate aerobic exercise, high-low intensity interval exercise, and breathing and strength training were effective in the treatment of CHF (39). Therefore, different forms, duration, intensity, and start time of exercise may result in different therapeutic effects in patients with CHF, and the mechanisms may also be different. As reported by Nunes et al. (40), aerobic continuous training (ACT) and aerobic interval training (AIT) were conducted in CHF rats. Both of the ACT and AIT were performed five times a week for 8 weeks, and the results showed that both the interval and continuous exercise could enhance aerobic capacity and hemodynamic function in CHF rats, while ACT showed higher $+\mathrm{dP} / \mathrm{dtmax}$ compared to AIT. However, the AIT was superior for improving maximal exercise capacity. Furthermore, Wisløff et al. (41) evaluated the effects of two different intensities of aerobic exercise training in $\mathrm{CHF}$ patients by 12 weeks of exercise training, and the results also supported the beneficial effect of aerobic exercise on CHF. In this present study, CHF rats performed continuous aerobic exercise training over an 8-week period, and the beneficial effects may be mediated via the miR-150-5p/ PI3K/Akt signaling pathway. Further experiments should be conducted to explore the mechanisms of aerobic exercise on rats at different stages of heart failure.

In conclusion, this study determined that aerobic exercise had a therapeutic effect on CHF rats, which was specifically manifested in inhibition of oxidative stress and inflammation, leading to reduced myocardial cell apoptosis and increased autophagy. Aerobic exercise reduced the expression of the lncRNA MALAT1 in rat myocardial tissue and increased the levels of miR-150-5p, and inhibited the downstream PI3K/Akt signaling pathway. These results demonstrated that aerobic exercise improved cardiac function through inhibition of the lncRNA MALAT1 in $\mathrm{CHF}$, and the potential mechanisms may be mediated via the miR-150-5p/PI3K/Akt signaling pathway. To elucidate the mechanism of aerobic exercise in heart failure may provide new targeted molecules for disease prevention and treatment.

\section{Acknowledgments}

Funding: This study was supported by the Beijing Municipal Natural Science Foundation (No. 7192242) and the Beijing Municipal Administration of Hospitals Clinical Medicine Development of Special Funding Support (No. ZYLX201835).

\section{Footnote}

Reporting Checklist: The authors have completed the ARRIVE reporting checklist. Available at http://dx.doi. org/10.21037/atm-20-8250

Data Sharing Statement: Available at http://dx.doi. org/10.21037/atm-20-8250

Conflicts of Interest: All authors have completed the ICMJE uniform disclosure form (available at http://dx.doi. org/10.21037/atm-20-8250). The authors have no conflicts of interest to declare.

Ethical Statement: The authors are accountable for all aspects of the work in ensuring that questions related to the accuracy or integrity of any part of the work are appropriately investigated and resolved. Experiments were performed under a project license (2019-101) granted by the Ethics Committee of Beijing Xiaotangshan Hospital, in compliance with Beijing Xiaotangshan Hospital guidelines for the care and use of animals.

Open Access Statement: This is an Open Access article distributed in accordance with the Creative Commons Attribution-NonCommercial-NoDerivs 4.0 International License (CC BY-NC-ND 4.0), which permits the noncommercial replication and distribution of the article with the strict proviso that no changes or edits are made and the original work is properly cited (including links to both the formal publication through the relevant DOI and the license). See: https://creativecommons.org/licenses/by-nc-nd/4.0/. 


\section{References}

1. Hoffman TM. Chronic Heart Failure. Pediatr Crit Care Med 2016;17:S119-123.

2. Dick SA, Epelman S. Chronic Heart Failure and Inflammation: What Do We Really Know? Circ Res 2016;119:159-76.

3. Špinar J, Spinarova L, Vitovec J. Pathophysiology, causes and epidemiology of chronic heart failure. Vnitr Lek 2018;64:834-8.

4. Tsutsui H, Kinugawa S, Matsushima S. Oxidative stress and heart failure. Am J Physiol Heart Circ Physiol 2011;301:H2181-90.

5. Costa S, Reina-Couto M, Albino-Teixeira A, et al. Statins and oxidative stress in chronic heart failure. Rev Port Cardiol 2016;35:41-57.

6. Arena R, Myers J, Forman DE, et al. Should highintensity-aerobic interval training become the clinical standard in heart failure? Heart Fail Rev 2013;18:95-105.

7. Alvarez P, Hannawi B, Guha A. Exercise And Heart Failure: Advancing Knowledge And Improving Care. Methodist Debakey Cardiovasc J 2016;12:110-5.

8. Vanhoutte PM, Shimokawa H, Feletou M, et al. Endothelial dysfunction and vascular disease - a 30th anniversary update. Acta Physiol (Oxf) 2017;219:22-96.

9. de Beer VJ, Bender SB, Taverne YJ, et al. Exercise limits the production of endothelin in the coronary vasculature. Am J Physiol Heart Circ Physiol 2011;300:H1950-9.

10. Fedewa MV, Hathaway ED, Ward-Ritacco CL. Effect of exercise training on $\mathrm{C}$ reactive protein: a systematic review and meta-analysis of randomised and non-randomised controlled trials. Br J Sports Med 2017;51:670-6.

11. Xie C, Zhang Y, Tran TD, et al. Irisin Controls Growth, Intracellular Ca2+ Signals, and Mitochondrial Thermogenesis in Cardiomyoblasts. PLoS One 2015;10:e0136816.

12. Quinn JJ, Chang HY. Unique features of long noncoding RNA biogenesis and function. Nat Rev Genet 2016;17:47-62.

13. Thomson DW, Dinger ME. Endogenous microRNA sponges: evidence and controversy. Nat Rev Genet 2016;17:272-83.

14. Li S, Mei Z, Hu HB, et al. The lncRNA MALAT1 contributes to non-small cell lung cancer development via modulating miR-124/STAT3 axis. J Cell Physiol 2018;233:6679-88.

15. Gong L, Chang H, Xu H. LncRNA MALAT1 knockdown alleviates oxygen-glucose deprivation and reperfusion induced cardiomyocyte apoptotic death by regulating miR122. Exp Mol Pathol 2019;111:104325.

16. Wang S, Yu W, Luo X, et al. MALAT1/miR-204/LC3II: A potential regulated axis of autophagy in myocardial ischemia-reperfusion injury. Int J Cardiol 2019;277:222.

17. Stølen T, Shi M, Wohlwend M, et al. Effect of exercise training on cardiac metabolism in rats with heart failure. Scand Cardiovasc J 2020;54:84-91.

18. Sties SW, Andreato LV, de Carvalho T, et al. Influence of exercise on oxidative stress in patients with heart failure. Heart Fail Rev 2018;23:225-35.

19. Nunes RB, Alves JP, Kessler LP, et al. Aerobic exercise improves the inflammatory profile correlated with cardiac remodeling and function in chronic heart failure rats. Clinics (Sao Paulo) 2013;68:876-82.

20. Radosinska J, Barancik M, Vrbjar N. Heart failure and role of circulating MMP-2 and MMP-9. Panminerva Med 2017;59:241-53.

21. Ho J, Yu J, Wong SH, et al. Autophagy in sepsis: Degradation into exhaustion? Autophagy 2016;12:1073-82.

22. $\mathrm{Hu} \mathrm{H}, \mathrm{Wu}$ J, Li D, et al. Knockdown of lncRNA MALAT1 attenuates acute myocardial infarction through miR-320Pten axis. Biomed Pharmacother 2018;106:738-46.

23. Yang $W, W u Z$, Yang $K$, et al. BMI1 promotes cardiac fibrosis in ischemia-induced heart failure via the PTENPI3K/Akt-mTOR signaling pathway. Am J Physiol Heart Circ Physiol 2019;316:H61-9.

24. Wang Y, Zhang Q. Long Noncoding RNA MALAT1 Knockdown Inhibits Proliferation, Migration, and Invasion and Promotes Apoptosis in Non-Small-Cell Lung Cancer Cells Through Regulating miR-515-3p/TRIM65 Axis. Cancer Biother Radiopharm 2020. [Epub ahead of print]. doi: 10.1089/cbr.2020.3730.

25. Ye M, Dong S, Hou H, et al. Oncogenic Role of Long Noncoding RNAMALAT1 in Thyroid Cancer Progression through Regulation of the miR-204/IGF2BP2/m6A-MYC Signaling. Mol Ther Nucleic Acids 2020;23:1-12.

26. Touma M, Kang X, Zhao Y, et al. Decoding the Long Noncoding RNA During Cardiac Maturation: A Roadmap for Functional Discovery. Circ Cardiovasc Genet 2016;9:395-407.

27. Zhang $\mathrm{M}, \mathrm{Gu} \mathrm{H}, \mathrm{Xu} W$, et al. Down-regulation of lncRNA MALAT1 reduces cardiomyocyte apoptosis and improves left ventricular function in diabetic rats. Int J Cardiol 2016;203:214-6.

28. Yan Y, Song D, Song X, et al. The role of lncRNA MALAT1 in cardiovascular disease. IUBMB Life 
2020;72:334-42.

29. Ou M, Zhao H, Ji G, et al. Long noncoding RNA MALAT1 contributes to pregnancy-induced hypertension development by enhancing oxidative stress and inflammation through the regulation of the miR-150-5p/ ET-1 axis. FASEB J 2020;34:6070-85.

30. Liu Q, Ma H, Sun X, et al. The regulatory ZFAS1/miR150/ST6GAL1 crosstalk modulates sialylation of EGFR via PI3K/Akt pathway in T-cell acute lymphoblastic leukemia. J Exp Clin Cancer Res 2019;38:199.

31. Wu SJ, Chen J, Wu B, et al. MicroRNA-150 enhances radiosensitivity by inhibiting the AKT pathway in NK/T cell lymphoma. J Exp Clin Cancer Res 2018;37:18.

32. Watanabe A, Tagawa H, Yamashita J, et al. The role of microRNA-150 as a tumor suppressor in malignant lymphoma. Leukemia 2011;25:1324-34.

33. Cattadori G, Segurini C, Picozzi A, et al. Exercise and heart failure: an update. ESC Heart Fail 2018;5:222-32.

34. Khan H, Kunutsor S, Rauramaa R, et al. Cardiorespiratory fitness and risk of heart failure: a population-based followup study. Eur J Heart Fail 2014;16:180-8.

35. Florido R, Ndumele CE, Kwak L, et al. Physical Activity, Obesity, and Subclinical Myocardial Damage. JACC Heart Fail 2017;5:377-84.

Cite this article as: $\mathrm{Hu} \mathrm{L}, \mathrm{Xu} \mathrm{YN}$, Wang Q, Liu MJ, Zhang P, Zhao LT, Liu F, Zhao DY, Pei HN, Yao XB, Hu HG. Aerobic exercise improves cardiac function in rats with chronic heart failure through inhibition of the long non-coding RNA metastasis-associated lung adenocarcinoma transcript 1 (MALAT1). Ann Transl Med 2021;9(4):340. doi: 10.21037/atm$20-8250$
36. Pandey A, Garg S, Khunger M, et al. Dose-Response Relationship Between Physical Activity and Risk of Heart Failure: A Meta-Analysis. Circulation 2015;132:1786-94.

37. Fleg JL, Cooper LS, Borlaug BA, et al. Exercise training as therapy for heart failure: current status and future directions. Circ Heart Fail 2015;8:209-20.

38. Cattadori G, Schmid JP, Brugger N, et al. Hemodynamic effects of exercise training in heart failure. J Card Fail 2011;17:916-22.

39. Vromen T, Kraal JJ, Kuiper J, et al. The influence of training characteristics on the effect of aerobic exercise training in patients with chronic heart failure: A metaregression analysis. Int J Cardiol 2016;208:120-7.

40. Nunes RB, Alves JP, Kessler LP, et al. Interval and continuous exercise enhances aerobic capacity and hemodynamic function in CHF rats. Braz J Phys Ther 2015;19:257-63.

41. Wisløff U, Støylen A, Loennechen JP, et al. Superior cardiovascular effect of aerobic interval training versus moderate continuous training in heart failure patients: a randomized study. Circulation 2007;115:3086-94.

(English Language Editor: J. Teoh) 